\title{
EL DERECHO Y LA INJUSTICIA EXTREMA ${ }^{1}$
}

LAW AND EXTREME INJUSTICE

Júlio Aguiar DE OLIVEIRA ${ }^{2}$

RESUMEN: mi propósito en este ensayo consiste en reflexionar en torno al argumento de la injusticia extrema (Fórmula de Radbruch), enfocándome tanto en las teorías no-positivistas del derecho de Gustav Radbruch y Robert Alexy como en los argumentos positivistas de H. L. A. Hart y Brian Bix, para así proponer algunos argumentos para defender la corrección de la tesis que reconoce la Fórmula de Radbruch como un elemento del concepto del derecho, pero no como una prescripción para la decisión judicial.

Palabras clave: Robert Alexy, Gustav Radbruch, Fórmula de Radbruch, Decisión judicial.

ABSTRACT: my purpose in this essay is to reflect on the argument of the extreme injustice (the Radbruch Formula), focusing on both the non-positivist theories of Gustav Radbruch and Robert Alexy's law and the positivist arguments of H. L. A. Hart and Brian Bix , in order to propose some arguments to defend the correctness of the thesis that recognizes the Radbruch formula as an element of the concept of law, but not as a prescription for judicial decision.

1 Traducción del portugués de Alejandro Nava Tovar, Profesor-Investigador en el Instituto Nacional de Ciencias Penales (INACIPE) y Profesor de Filosofía del Derecho en el Posgrado en Derecho de la UNAM. Miembro del Sistema Nacional de Investigadores (SNI 1). ORCID: <https://orcid.org/0000-0002-5770-5998>.

2 Profesor-investigador de la Universidade Federal de Ouro Preto y de la Pontifícia Universidade Católica de Minas Gerais (Brasil). Una primera versión de este ensayo fue escrita durante una estancia posdoctoral en la Universidad de Kiel (Alemania) y presentada en el Seminario Superior de la Cátedra de Filosofía del Derecho y Derecho Público de la Universidad de Kiel, en julio de 2013. Mis agradecimientos a los profesores Robert Alexy y Stanley L. Paulson, a la Sra. Andrea Neisius y a mi colega Alejandro Nava Tovar. También quiero agradecer a la CAPES, a la Universidade Federal de Ouro Preto y a la Pontifícia Universidade Católica de Minas Gerais, por su apoyo durante la realización de mi estancia posdoctoral.

Fecha de recepción: 13 de diciembre de 2018; fecha de aprobación: 13 de marzo de 2019. 
Keywords: Robert Alexy, Gustav Radbruch, Radbruch Formula, Judicial Decision.

Sumario: I. Introducción II. La Fórmula de Radbruch en el contexto de la filosofía del derecho de Radbruch: la especificidad de la normatividad del derecho III. El papel de la Fórmula de Radbruch en el concepto de derecho de Alexy IV. La Fórmula de Radbruch según la tesis de Brian Bix V. Conclusión VI. Bibliografía

\section{INTRODUCCIÓN}

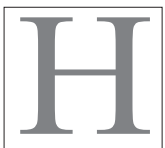

ay dos funciones posibles para la Fórmula de Radbruch, sintetizada por Robert Alexy mediante la expresión "la injusticia extrema no es derecho". La Fórmula de Radbruch puede ser tomada, en primer lugar, como una propuesta conceptual sobre la naturaleza del derecho (más específicamente, como un elemento del concepto del derecho) y, en segundo lugar, puede ser tomada como una prescripción para la decisión judicial. Al considerarse estas dos funciones como puntos de partida, pueden presentarse cuatro tesis diferentes. Primera: la Fórmula de Radbruch es comprendida como una propuesta conceptual sobre la naturaleza del derecho y también como una prescripción para la decisión judicial. Segunda: la Fórmula de Radbruch es rechazada como una propuesta conceptual sobre la naturaleza del derecho y también como una prescripción para la decisión judicial. Tercera: la Fórmula de Radbruch es rechazada como una propuesta conceptual sobre la naturaleza del derecho, pero es admitida como una prescripción para la decisión judicial. Cuarta, y aquí agotamos las posibilidades: la Fórmula de Radbruch es admitida como una propuesta conceptual sobre la naturaleza del derecho, pero es rechazada como una prescripción para la decisión judicial (estas posibilidades pueden visualizarse fácilmente en la tabla incluida al final de este texto). Mi propósito en este ensayo consiste en reflexionar en torno al argumento de la injusticia extrema (Fórmula de Radbruch), enfocándo- 
me en las teorías del derecho de Gustav Radbruch y Alexy, y así proponer algunos argumentos para defender la corrección de la última de las cuatro tesis presentadas, a saber, la tesis que reconoce la fórmula Radbruch como un elemento del concepto del derecho, pero no como una prescripción para la decisión judicial. En este sentido, empezaré con el tratamiento de la Fórmula de Radbruch en el contexto mismo de la filosofía del derecho de Radbruch. Mi objetivo principal en este momento es mostrar que la Fórmula de Radbruch no debe interpretarse como la prueba de una supuesta ruptura de Radbruch, después de la Segunda Guerra Mundial, con su también supuesta afiliación original con el positivismo jurídico. A diferencia de los que defienden la tesis de la existencia de una especie de conversión de Radbruch que va del positivismo al iusnaturalismo (o no-positivismo), considero que Radbruch desarrolla una teoría del derecho no-positivista consistente a lo largo de toda su obra. Esta comprensión refuerza el entendimiento de que, en la teoría del derecho de Radbruch, la Fórmula de Radbruch tiene, sobre todo, el significado de un elemento del concepto del derecho. Ella es, en esencia, un aspecto central de la respuesta de Radbruch a la cuestión de la normatividad específica del derecho. Luego paso al análisis de la Fórmula de Radbruch en la teoría del derecho de Alexy. En este punto, sostengo que, aunque en Alexy la Fórmula de Radbruch desempeñe tanto la función de elemento del concepto de derecho, así como una función de prescripción para la decisión judicial, sería mejor, teniendo en cuenta en particular los argumentos de la democracia y de la seguridad jurídica, que no tuviera la función de prescripción para la decisión judicial. Por último, examino la tesis propuesta por Brian Bix, quien admite la validez de la Fórmula de Radbruch como prescripción para la decisión judicial, pero le niega la función de un elemento del concepto del derecho. Sobre este último tópico, mi intención es analizar y refutar los dos argumentos propuestos por Bix para sustentar sus tesis respectivas: el argumento del contexto y el argumento de la perspectiva. 
II. LA Fórmula de Radbruch EN EL CONTEXTO DE LA FILOSOFÍA DEL DERECHO DE RADBRUCH: LA ESPECIFICIDAD DE LA NORMATIVIDAD DEL DERECHO

La naturaleza conceptual de la Fórmula de Radbruch, entendida como un elemento fundamental de la normatividad específica del derecho, puede verse claramente cuando es analizada la Fórmula de Radbruch en el contexto de la crítica de Radbruch al positivismo jurídico. Según Radbruch:

El positivismo cree haber demostrado la validez de las leyes cuando estas leyes tienen el poder suficiente para ser establecidas. Pero mientras el poder puede fundamentar una obligación, jamás podrá fundamentar un deber o la validez jurídica. Más bien, la validez jurídica y la obligación están fundamentadas en un valor, el cual es inherente a la ley. ${ }^{3}$

En esta sección puede verse la razón básica por la que Radbruch se niega a aceptar al positivismo jurídico como una teoría adecuada del derecho. Radbruch se opone al positivismo jurídico porque éste no es capaz de explicar el deber de la obligación. ${ }^{4}$ Este es el punto. ${ }^{5}$

3 Radbruch, Gustav, "Arbitrariedad legal y derecho supralegal", en, Paulson, Stanley, L, La filosofía del derecho de Gustav Radbruch, Madrid, Marcial Pons, 2019, p. 219.

4 "La moral apenas puede fundamentar la fuerza de la obligación del derecho. De los preceptos del derecho como imperativos, como manifestaciones de la voluntad, se puede, como se ha mostrado, derivar un deber (Müssen), pero nunca un deber ser (Sollen). Sólo puede hablarse de normas jurídicas, de deber ser jurídico, de validez jurídica y de obligaciones jurídicas cuando el imperativo jurídico esté acompañado por la conciencia individual con la fuerza de la obligación moral." Radbruch, Gustav, Rechtsphilosophie, Heidelberg, C. F. Müller, 2003, p. 47.

5 Vale la pena señalar aquí, conforme lo observa Paulson, que no es acertado inferir, a partir de los escritos de Radbruch publicados después de la Segunda Guerra Mundial, algo así como una defensa de una teoría causal que asigna la responsabilidad al positivismo por la catástrofe nazi. Para Radbruch el positivismo jurídico debe ser rechazado como una teoría del derecho no porque sea una teoría nazi del derecho (lo que simplemente no se ajusta a los hechos), sino porque no 
La Fórmula de Radbruch es un intento de abordar específicamente este problema. Este problema puede ser expuesto por la pregunta: ¿cómo debe fijarse el aspecto normativo específico del derecho positivo? La respuesta que Radbruch sugiere para esta pregunta aparece en su forma completa en sus escritos posteriores a la Segunda Guerra Mundial, pero ella ya había aparecido, en parte, en sus escritos antes de esta guerra. Esta respuesta incluye la afirmación de un principio regulativo - la idea del derecho - en el núcleo de la normatividad del derecho.

La manera en que yo entiendo en su configuración final este principio regulativo, situado en el núcleo de la normatividad del derecho, se encuentra desarrollado en dos principios relacionados entre sí: en primer lugar, como un principio regulativo del derecho y, en segundo lugar, como un principio regulativo en cuanto expresión del límite inferior de la manifestación del principio regulativo ideal para conferirle a la norma jurídica su carácter normativo específico, necesario para la constitución de su naturaleza jurídica específica, que llegará a ser conocido, más tarde, como la Fórmula de Radbruch.

Estos dos elementos definen el horizonte de la normatividad específica del derecho positivo, lo que, a su vez, es un elemento esencial del concepto de derecho. Tanto en los escritos anteriores como en los escritos posteriores de la Segunda Guerra Mundial Radbruch ofrece una respuesta única al problema de la normatividad específica del derecho positivo, una respuesta que, como se ha visto, constituye la revelación de un principio regulativo que se desarrollará, por una parte, como la idea del derecho y, por otra parte,

es capaz, como se ha visto, de explicar el deber de la obligación. Cfr. Paulson, Stanley, L., "Lon Fuller, Gustav Radbruch y las tesis positivistas", en, Paulson, Stanley L., La filosofía del derecho de Gustav Radbruch, Madrid, Marcial Pons, 2019, pp. 53-100. Acerca del error de atribuirle al positivismo la función de base teórica para la concepción nazi del derecho, cfr. Rüthers, Bernd, Die unbegrenzte Auslegung, Tubinga, Mohr Siebeck, 2012, p. 99. 
como la Fórmula de Radbruch, la cual sostendrá que una injusticia extrema no es derecho, lo cual de esta forma marcará los límites de la juridicidad.

Sin embargo, la novedad que aparece en los escritos posteriores a la Segunda Guerra Mundial es la búsqueda de los aspectos sustantivos de la injusticia extrema. En Gesetzliches Unrecht und übergesetzliches Recht (Arbitrariedad legal y derecho supralegal) de 1946, al tiempo que reconoce la imposibilidad de trazar una línea de demarcación exacta entre una injusticia extrema, que lleva a la negación misma del carácter jurídico de una norma positiva, y una injusticia no extrema, que no es todavía suficiente para la negación de la naturaleza jurídica de una norma positiva, Radbruch propone las siguientes aproximaciones: a) una ley que desprecia los principios básicos del derecho natural expresado en las declaraciones de derechos humanos y civiles, no es derecho; b) una ley que desprecia la justicia como fin del derecho positivo, particularmente a través de una traición de la igualdad, no es derecho; c) una ley que niega a los seres humanos sus derechos humanos, especialmente a través de la negación del reconocimiento de su condición humana, no es derecho; d) una ley que impone la misma pena a muy diferentes clases de delitos, sin atención a la gravedad específica de cada uno de los delitos, no es derecho. Estos contenidos no sólo son injustos, son extremadamente injustos, y por lo tanto llevan a la negación de la naturaleza jurídica de la ley en la cual se presenten. ${ }^{6}$

La atención de Radbruch para la definición de los aspectos sustanciales de la injusticia extrema, sin embargo, no implica el abandono de la seguridad jurídica en la forma de abandono de la doctrina de la vinculación del juez al derecho positivo. En este punto en particular, mi interpretación es diferente de la interpretación de Stanley L. Paulson, una vez que, de acuerdo con Paulson: “[E]n sus escritos

6 Cfr. Radbruch, Gustav, "Arbitrariedad legal y derecho supralegal", en, Paulson, Stanley, L, La filosofia del derecho de Gustav Radbruch, Madrid, Marcial Pons, 2019, pp. 213-225. 
de posguerra, Radbruch pone el criterio basal [la idea del derecho] a trabajar, liberándolo de las ataduras impuestas por la doctrina de la vinculación del juez al derecho positivo."7 Por lo tanto, Paulson entiende que Radbruch en sus escritos de posguerra rompió con la doctrina de la vinculación del juez al derecho positivo.

La cuestión aquí es la definición de lo que significa en la práctica una teoría no-positivista. Si puesta en práctica significa necesariamente tener la Fórmula de Radbruch como una prescripción para la decisión judicial, rechazando así la doctrina de la vinculación del juez a la ley, entonces, de hecho, la teoría no-positivista de Radbruch sólo puede interpretarse como si hubiera sido puesta en práctica a partir de sus escritos publicados después de la Segunda Guerra Mundial. Sin embargo, creo que es posible que la teoría no-positivista Radbruch pueda implementarse sin tener que ser aplicada como una prescripción para la decisión judicial. Creo que es posible argumentar que la Fórmula de Radbruch es, de hecho, un elemento esencial del concepto del derecho y, al mismo tiempo, que ella no puede ser entendida como una prescripción para la decisión judicial. La combinación de estas dos proposiciones, sin embargo, no quiere decir, y esto me parece ser un resultado práctico, que los jueces están obligados a aplicar las leyes extremadamente injustas (de hecho, los jueces no están obligados a aplicar las leyes extremadamente injustas porque una injusticia extrema no es derecho), ni que no deben ser culpables los jueces por la aplicación de una ley extremadamente injusta. Por lo tanto, la Fórmula de Radbruch no cancela ni debilita la doctrina de la vinculación del juez a la ley, ya que no le da al juez el poder de decidir como juez, qué leyes son derecho y qué leyes no son derecho en virtud de su contenido extremadamente injusto.

7 Paulson, Stanley, L., "On the Background and Significance of Gustav Radbruch's Post-War Papers", en, Oxford Fournal of Legal Studies, vol. 26, No. 1 (2006): 17-40. p. 38 . 
Es necesario, sin embargo, dejar claro que el conflicto entre una ley con un contenido extremadamente injusto (es decir, una injusticia extrema) y la vinculación del juez a la ley es un conflicto necesariamente trágico. Frente a este conflicto, un juez tiene el deber de resistir a la injusticia legal. Este deber de resistencia, como lo comprende Radbruch, se deriva del hecho de que el "ethos del juez debe ser dirigido a la justicia a cualquier precio, incluso al precio de su propia vida." 8 ste deber de resistencia, sin embargo, no significa que el juez puede, o debe, declarar la nulidad de una ley sobre la base de su contenido extremadamente injusto. La cuestión aquí no es una cuestión de inconstitucionalidad de la ley. Esto no es un problema que puede resolverse dentro de los límites de la interpretación de las normas constitucionales. Ante una injusticia extrema, no hay posibilidad de respuesta por parte de la interpretación de la Constitución (en caso de ser posible una respuesta dentro de los marcos de la interpretación de la Constitución, o mejor, si el presunto contenido de una ley extremadamente injusta puede llevar a la cancelación de la misma ley debido a su inconstitucionalidad, entonces esta ley no puede ser tomada como un ejemplo de injusticia extrema en sentido estricto). Por lo tanto, entiendo que frente a un caso de injusticia extrema en sentido estricto, el concepto no-positivista de derecho de Radbruch no deja al juez otra alternativa que renunciar a su cargo. No hay solución, desde el punto de vista de la interpretación del derecho, para este problema. Ante una injusticia extrema, el juez se encuentra en la situación de ser, al mismo tiempo, tanto la persona que tiene el deber de conciencia de considerar todas las leyes adecuadamente promulgadas como leyes válidas, es decir, como derecho, como también la persona que tiene un deber de conciencia de considerar como una injusticia extrema una ley adecuadamente promulgada con un contenido extremadamente

8 Radbruch, Gustav, "Arbitrariedad legal y derecho supralegal”, en, Paulson, Stanley, L, La filosofia del derecho de Gustav Radbruch, Madrid, Marcial Pons, 2019, p. 224. 
injusto. En este caso, es como si el juez y el criminal por convicción se encontrasen fusionados en una sola persona. Radbruch señala:

(...) ante un juez, obligado por su conciencia a considerar todo el derecho establecido como derecho vigente puede ponerse un acusado, también obligado por su conciencia a considerar como no válido, aunque establecido, el derecho injusto o inadecuado a su fin. El derecho puede hacer valer su poder frente a él, pero nunca demostrar su validez. Este caso de "delincuente por convicción" se evidencia como un caso realmente trágico precisamente porque para él no hay solución. ${ }^{9}$

La confrontación de un juez con una injusticia extrema también puede ocurrir de otra manera. Un juez puede confrontarse con la afirmación de que una determinada ley debidamente establecida y eficaz, parte de un orden legal del pasado, nunca poseyó, debido a la injusticia extrema de su contenido, un carácter legal. Este fue, de hecho, el argumento presentado contra el Decreto Onceavo de la Ley de Ciudadanía del Reich del 25 de noviembre 1941. ${ }^{\circ}$ Una vez más, no creo que la Fórmula de Radbruch otorga al juez la facultad de declarar que una ley, correctamente establecida y eficaz, no tenía carácter legal debido a la extrema injusticia de su contenido. Este tipo de declaración presupone un poder que no se le puede asignar a un juez (o incluso a una Corte Suprema de Justicia) sin que se ponga en riesgo no sólo la seguridad jurídica sino también la dignidad de la ley, porque, como observa Radbruch, "la ley tiene valor no sólo como una precipitación de la justicia, sino también como una garantía de la seguridad jurídica y es predominantemente en este último sentido que se le ha encomendado al juez". ${ }^{11}$ Este poder debe residir únicamente en el órgano legislativo. Al tener en cuenta

9 Radbruch, Gustav, Rechtsphilosophie, Heidelberg, C. F. Müller, 2003, p. 85.

1011 Veordnung zum Reichsbürgergesetz vom 25. November 1941.

11 Radbruch, Gustav, Rechtsphilosophie, Heidelberg, G. F. Müller, 2003, p. 182. 
esta situación particular, el juez debe reconocer que sólo los que tienen el poder de hacer leyes pueden tener la facultad de declarar que una ley debidamente promulgada no es derecho debido a la injusticia extrema de su contenido.

¿Esto significa que mi interpretación de la Fórmula de Radbruch conduce a su autor al positivismo jurídico? ¿O acaso, teniendo en cuenta la teoría ampliamente aceptada, aunque errónea, de que Radbruch experimentó una conversión del positivismo jurídico al iusnaturalismo natural después de la Segunda Guerra Mundial, esto significa que mi interpretación lo conduce de nuevo al lado del positivismo jurídico? No lo creo. De hecho, siguiendo por el camino abierto por Paulson, Ralf Dreier y otros, estoy convencido de que Radbruch nunca fue un iuspositivista. ${ }^{12}$ Por lo tanto, no me parece correcto decir que ha sido objeto de una especie de conversión del positivismo al iusnaturalismo. Él no fue un juspositivista antes y, por esa razón, simplemente no podía convertirse en un no-positivista después de la guerra. Un criterio basal neokantiano, como Paulson lo designó, ya se muestra desarrollado como un principio regulativo en el concepto de derecho de Radbruch en sus escritos anteriores a la guerra y se mantiene presente - aún más notablemente- en sus escritos publicados después de la Segunda Guerra Mundial. La Fórmula de Radbruch es esencialmente el resultado del desarrollo de esto criterio basal neokantiano. Por lo tanto, parece claro que Radbruch sostiene que hay una conexión necesaria entre el derecho y la moral. Esta conexión ya la sostiene Radbruch en su trabajo an-

12 En este sentido cfr., Paulson, Stanley L., "Ein Ewiger Mythos: Gustav Radbruch als Rechtspositivist - Teil 1" Juristen-Zeitung, 2008: 105-115; "Radbruch on Unjust Laws: Competing Earlier and Later Views?", en, Oxford Fournal of Legal Studies, vol. 15, No. 3, 1995: 489-500; "On the Background and Significance of Gustav Radbruch's Post-War Papers", en, Oxford Fournal of Legal Studies, vol. 26, No. 1 (2006): 17-40; Kaufmann, Arthur, Gustav Radbruch-Rechtsdenker, Philosoph, Sozialdemokrat; Münich, Piper, 1987; Wolf, Erik, "Umbruch oder Entwicklung in Gustav Radbruchs Rechtsphilosophie?", en, Archiv für Rechts und Sozialphilosophie 45, 1959, 481-503. 
terior a la guerra y la seguirá sosteniendo en su trabajo posterior a la guerra, lo que lo pone, antes y después de la guerra, en el campo no-positivista.

Uno también puede preguntarse si esta interpretación del concepto de derecho de Radbruch lo conduce hacia el ámbito del no-positivismo superincluyente (bajo la clasificación propuesta por Alexy). ${ }^{13}$ Una vez más, entiendo que este no es el caso. La tesis de que la Fórmula de Radbruch no debe ser tomada como una prescripción para la decisión judicial no implica la negación de la existencia de una conexión clasificatoria entre el derecho y la moral. Aunque defiendo la tesis de que un juez (poder judicial) no es competente para declarar que una norma concreta regularmente promulgada y socialmente eficaz no es derecho debido a la extrema injusticia de su contenido, esto no conduce a ningún cambio en el estatus de no-derecho de dicha ley. Una injusticia extrema no es derecho, aunque el juez (poder judicial) no sea competente para hacer tal declaración. Esto significa, en términos prácticos, que esta ley no debe observarse ni cumplirse, lo cual lleva al reconocimiento de las consecuencias prácticas de la conexión entre el derecho y la moral. ${ }^{14}$

Al tener en cuenta la teoría del derecho de Radbruch en su integridad (y prestando atención a la importancia de la conservación de una unidad lógica a lo largo del desarrollo de su obra), parece claro que la negación de la función de la Fórmula de Radbruch en cuanto prescripción para la decisión judicial es una condición bien justificada para una interpretación coherente de la teoría jurídica

13 Cfr. Alexy, Robert, "The Dual Nature of Law", en, Ratio Furis, Vol. 23, no. 2, 2010 , p. 176.

14 Según Paulson, sin embargo, una teoría no-positivista del derecho no es puesta en práctica si no se le da al juez la facultad de aplicar la Fórmula de Radbruch como una prescripción para la decisión judicial. Cfr. Paulson, Stanley, L., "On the Background and Significance of Gustav Radbruch's Post-War Papers", p. 32. 
de Radbruch. ${ }^{15}$ En la siguiente sección, voy a examinar el papel de la Fórmula de Radbruch en el concepto de derecho propuesto por Alexy.

iil. El papel de la fórmula de Radbruch en el concepto DE DEREcho de Alexy

La esencia de la Fórmula de Radbruch en la teoría del derecho de Alexy consiste en ser un elemento del concepto de derecho. La función de esta fórmula, así como se observa en la teoría de Radbruch, es la de fundamentar la normatividad específica del derecho. Alexy identifica dos elementos específicos en el terreno de la normatividad del derecho: la pretensión de corrección y el argumento de la injusticia. Estos dos elementos pueden interpretarse, por analogía, como los dos elementos en los cuales se desarrollan el principio regulativo en la teoría de Radbruch: la idea del derecho y la injusticia extrema. El carácter de estos elementos como elementos de la normatividad específica del derecho no se explica sólo por el hecho de que la pretensión de corrección se toma en conjunto

15 Mucho más debería decirse con la finalidad de defender correctamente la tesis de una teoría del derecho coherente e integralmente no-positivista en Radbruch, especialmente teniendo en cuenta la dificultad de la cuestión del "relativismo de Radbruch". A partir de la propuesta de una distinción entre valores (Werte) y juicios de valor (Werturteilen), Marc André Wiegand apoya la tesis de que una reivindicación tradicional de la irracionalidad de los valores en Radbruch debería interpretarse como dirigida no a los valores, sino a los juicios valor, una interpretación más adecuada respecto al concepto de derecho de Radbruch y, también, a su neokantismo. En este sentido, cfr. Wiegand, Marc, Unrichtiges Recht, Tubinga, Mohr Siebeck, 2004, p. 30-31. Especialmente en relación al neokantismo de Radbruch, cfr. Paulson, "On the Background and Significance of Gustav Radbruch's Post-War Papers", p. 29-32. También, para considerar el "relativismo de Radbruch" con el fin de ponerlo en consonancia con un no-positivismo coherente, cfr. Von der Pfordten, Dietmar, "Radbruch as an Affirmative Holist. On the Question of what Ought to Be Preserved of his Philosophy", Ratio Iuris, 21, 2008, p. 399. 
con el argumento de la injusticia, sino también por el hecho de que tanto la pretensión de corrección como también el argumento de la injusticia están conectados con los elementos clásicos de la promulgación de acuerdo con el ordenamiento y de la eficacia social. En el concepto de derecho de Alexy, la Fórmula de Radbruch es la expresión de la normatividad específica del derecho. Ella es la expresión misma de la teoría de la tesis de la conexión.

Como ya se ha dicho, la Fórmula de Radbruch puede y debe entenderse como un elemento de una proposición conceptual acerca del derecho y no como una prescripción para la decisión judicial. La pregunta ahora es: ¿hay buenas razones para negarle a la Fórmula de Radbruch la función de prescripción para la decisión judicial, incluso en el contexto del concepto no-positivista de derecho de Alexy? Creo que hay dos razones aquí: la democracia y la seguridad jurídica.

La objeción a un concepto no-positivista del derecho basada en la democracia se basa en la idea de que, según Alexy, "el concepto no-positivista de derecho lleva consigo el riesgo de que el juez, apelando a la justicia, va a oponerse a las decisiones del legislador democráticamente legitimado". ${ }^{16}$ En defensa de un concepto no-positivista de derecho, en el cual la Fórmula de Radbruch también posee la función de una prescripción para la decisión judicial, Alexy afirma:

Esta objeción pierde su fuerza si se considera que el concepto no-positivista de derecho sólo permite la eliminación del carácter legal en casos de extrema injusticia. Sólo toma efecto en un contexto nuclear. El control de la Corte Constitucional sobre violaciones de los derechos constitucionales fundamentales en Estados democráticos va mucho más allá de ese ámbito nuclear. Por lo tanto, para presentar un argumento de la democracia o de la división de poderes en contra de la tesis débil de la vinculación defendido aquí, tendría

16 Alexy, Robert, Begriff und Geltung des Rechts, Múnich, Karl Alber, 1992, p. 97. 
que rechazarse todo control de constitucionalidad de compromiso del legislador con los derechos fundamentales. ${ }^{17}$

Una vez que un juez en un Estado democrático de derecho establece que una ley particular que se ha establecido correctamente no es (o no era) derecho en virtud de su contenido extremadamente injusto, esto constituye, en mi opinión, una violación de la democracia. Tal decisión es incompatible con la democracia. Es cierto, como señala Alexy, que "el control de la Corte Constitucional sobre violaciones de los derechos constitucionales fundamentales en Estados democráticos va mucho más allá"18 del umbral de la extrema injusticia. Si es así, sin embargo, la conclusión debe ser la afirmación de la imposibilidad de que se produzca una injusticia extrema en un Estado democrático de derecho. Sin embargo, si se acepta la posibilidad de aparición de una injusticia extrema en un Estado democrático de derecho, entonces debe aceptarse también que, por alguna razón, el control de la Corte Constitucional en relación con violaciones de los derechos fundamentales no puede evitar la injusticia extrema. En este sentido, la crítica que surge del argumento de la democracia contra el uso de la Fórmula de Radbruch como prescripción para la decisión judicial no depende del rechazo de cualquier conexión del legislador con los derechos fundamentales que pueda someterse al control judicial, y parece ser el caso de que un concepto no-positivista del derecho, en el cual la Fórmula de Radbruch desempeña tal función de prescripción para la decisión judicial, se encuentre, de hecho, en contradicción con la democracia.

El otro argumento en contra de la utilización de la Fórmula de Radbruch como prescripción para la decisión judicial es la seguridad jurídica. Según Alexy, "un argumento contra el concepto no-positivista de derecho señala que este concepto pone en peligro

\footnotetext{
17 Alexy, Robert, op. cit., p. 97.

18 Alexy, Robert, op. cit., p. 97.
} 
la seguridad jurídica." 19 Alexy, sin embargo, entiende que, debido a la aplicación del principio del reconocimiento, puede preservarse la seguridad jurídica sin rechazar el empleo de la Fórmula de Radbruch como prescripción para la decisión judicial. Alexy dice:

Si existen juicios de justicia fundamentables racionalmente, puede entonces decirse que quien, sobre la base de una fundamentación racional, ve que una acción es injusta reconoce esto. Ahora bien, vale el siguiente enunciado: cuanto más extrema sea la injusticia, tanto más seguro es su conocimiento. Este enunciado vincula el aspecto material con el epistemológico. ${ }^{20}$

Aunque la definición de una injusticia extrema pueda orientarse hoy por los derechos humanos, el reconocimiento de la injusticia extrema no es necesariamente sencillo. La injusticia extrema del Onceavo Decreto de la Ley de Ciudadanía del Reich, por ejemplo, no fue inmediatamente reconocida por los jueces y otras autoridades judiciales y administrativas. Ésta tomó tiempo y un cambio político radical para que se pudiera tener en cuenta la injusticia extrema de este decreto.

La razón de esta dificultad se debe, al menos en parte, al hecho de que la injusticia extrema se expresa necesariamente en la forma de una ley establecida correctamente. Una injusticia extrema no es simplemente un terrible acto de injusticia, o un crimen, o una violación de los derechos humanos. Injusticia extrema - en sentido estricto, es decir, injusticia extrema como un elemento del concepto de derecho - sólo puede presentarse en la forma de una ley debidamente establecida con un contenido extremadamente injusto. El hecho de que este contenido extremadamente injusto se encuentre en una ley debidamente establecida es exactamente lo que hace que sea difícil reconocer una injusticia extrema. Pero esta dificultad, al mismo tiempo, es evidencia de la idoneidad de la Fórmula de Ra-

\footnotetext{
19 Alexy, Robert, op. cit., p. 90.

20 Alexy, Robert, op. cit., p. 91.
} 
dbruch como un elemento del concepto de derecho. Si el derecho fuera un sistema de normas neutrales desde el punto de vista moral, sería más fácil reconocer una injusticia extrema. Tan pronto no exista algún valor moral específico del derecho, los valores morales, solamente reflejados por el derecho, brillarían como estrellas en la noche en el desierto, no oscurecidos por las luces de la ciudad. Esto explica por qué la propuesta de H. L. A. Hart suena tan razonable a primera vista. ${ }^{21} \mathrm{Al}$ eliminar del concepto de derecho su normatividad específica, que es necesariamente un elemento de carácter moral, parece que se vuelve más fácil la identificación de los casos en los que el derecho es simplemente el vehículo de la injusticia y, por lo tanto, parece que se vuelve más fácil resistir a las injusticias legales. Retirar la normatividad específica del derecho del conjunto de los elementos del concepto de derecho parece ser no sólo correcto desde el punto de vista epistemológico, sino también correcto desde el punto de vista moral. Habría entonces una razón moral para la tesis de la separación entre derecho y moral. Una razón moral, por lo tanto, para el propio positivismo. ${ }^{22}$

21 Cfr. Hart, H. L. A., «Positivism and the Separation of Law and Morals», en, Harvard Law Review 7 1, 1957-1958, p. 620.

22 "En tanto que los seres humanos puedan ganar suficiente cooperación de algunos otros para que puedan dominar a los demás, ellos van a utilizar a las formas del derecho como uno de sus instrumentos. Los hombres malvados promulgarán normas perversas que otros cumplirán. Lo que sin duda es más que necesario con el fin de hacer que los hombres lúcidos enfrenten el abuso del poder oficial, es preservar el sentido de que la certificación de algo como legalmente válido no es concluyente sobre la cuestión de la obediencia, y que, por más grande que sea la aura de majestad o autoridad que el sistema oficial podría tener, sus demandas deben al final presentarse bajo el escrutinio moral. Este sentido, que hay algo que está fuera del sistema oficial haciendo referencia a lo que en última instancia el individuo tiene que resolver respecto al problema de la obediencia, seguramente es más probable que se mantenga vivo con quienes estamos acostumbrados a pensar que las normas jurídicas pueden ser injustas, que entre aquellos que piensan que no hay nada injusto en cualquier lugar que pueda tener el estatus de derecho". Hart, H. L. A., The Concept of Law, Oxford, Clarendon Press, 1998, p. 210. 
No obstante, el problema con esta tesis es el mismo que enfrentamos al intentar tirar al bebé con el agua del baño. Nadie pone en duda el hecho de que la iluminación nocturna de las ciudades afecta a la observación de las estrellas, pero no es una opción apagar las luces de la ciudad para observar mejor las estrellas. Del mismo modo, es imposible eliminar la normatividad específica del derecho sin hacer desaparecer el derecho mismo. Lo que hace el derecho vinculante es parte de lo que es el derecho.

Puede que no sea fácil, por tanto, el reconocimiento de una injusticia extrema. Esto, sin embargo, no parece ser un argumento a favor de la teoría de la separación (Trennungsthese), según lo propuesto por Hart, pero, por otra parte, a mí me parece un argumento en contra del uso de la Fórmula de Radbruch como una prescripción para la decisión judicial, porque si la injusticia extrema no tiene necesariamente la marca de la evidencia, entonces no parece aceptable afirmar que la Fórmula de Radbruch entendida como prescripción para la decisión judicial sólo conduce a una pérdida mínima de la seguridad jurídica.

iv. La fórmula de Radbruch según La tesis de Brian Bix

Brian Bix afirma que la Fórmula de Radbruch tiene la función de prescripción para la decisión judicial. Según Bix, "sería más benevolente leer a la(s) fórmula(s) de Radbruch como prescripciones para la decisión judicial que en vez de leerlas como proposiciones descriptivas, conceptuales o analíticas respecto a la naturaleza del derecho." ${ }^{23}$ En este sentido, Bix presenta dos argumentos: (1) el argumento del contexto y (2) el argumento de la perspectiva. Con estos dos argumentos, Bix tiene la intención de mostrar que la Fórmula de Radbruch no está dirigida para resolver el problema de la naturaleza del derecho. En primer lugar, porque ella fue utilizada

23 Bix, Brian, «Radbruch's Formula and Conceptual Analysis», en, American Fournal of Furisprudence, 56, 2008, p. 57. 
como una prescripción para la decisión judicial (lo que llamo el argumento de contexto). En segundo lugar, porque ella fracasa cuando uno la toma como un elemento del concepto de derecho (lo que llamo el argumento de la perspectiva). En consecuencia, de acuerdo con Bix, la Fórmula de Radbruch no funciona como una crítica hacia el positivismo jurídico. Al no estar dirigida a las cuestiones sobre la naturaleza del derecho, Bix asume que la Fórmula de Radbruch debe entenderse como una prescripción para la decisión judicial. Sin embargo, esto no puede ser más que una conjetura, una vez que Bix no ofrece ningún argumento preciso para apoyar esta tesis.

El primer argumento de Bix para demostrar que la Fórmula de Radbruch tiene esencialmente el significado de una prescripción para la decisión judicial consiste en explorar el contexto en el que han aparecido los escritos de Radbruch, y, más tarde, los de Alexy, directamente relacionados con la tesis que vino a ser conocida como la Fórmula de Radbruch.

Desde una perspectiva superficial, no hay duda de que, sea lo que sea, la Fórmula de Radbruch (o las fórmulas Radbruch) en realidad funcionan como instrucciones a los jueces sobre la forma de resolver los casos. Como se mencionó anteriormente, la decisión del tribunal (por los tribunales de Alemania Occidental en relación con los actos supuestamente realizados bajo la autorización de las leyes nazis) constituyen el contexto en el que Radbruch presenta su fórmula (o fórmulas) en sus artículos de posguerra. Decisiones similares adoptadas por los tribunales de la Alemania reunificada, evaluando actos supuestamente realizados bajo la autorización del derecho de la Alemania oriental, forman el contexto para algunas de las discusiones de Alexy acerca de su versión de la Fórmula de Radbruch. ${ }^{24}$

Estoy de acuerdo con Bix en que la Fórmula de Radbruch fue aplicada por los tribunales de Alemania Occidental y, también, más tarde por los tribunales de la Alemania reunificada, pero eso no

24 Bix, Brian, op. cit., p. 52. 
significa necesariamente que ella deba ser definida como una prescripción para las decisiones judiciales. Un pie de cabra se puede utilizar para abrir una puerta y, de hecho, pies de cabra se utilizan ocasionalmente para abrir las puertas. Sin embargo, este hecho no funciona como argumento para decir que un pie de cabra debe definirse fundamentalmente como una clave. Lo mismo puede decirse de la Fórmula de Radbruch. Del hecho de que se le utilizó como una prescripción para las decisiones judiciales no se sigue que ella deba ser definida como una prescripción para la decisión judicial. El argumento del contexto propuesto por Bix simplemente no justifica tal conclusión.

Por otra parte, teniendo en cuenta los mismos textos en los que la reflexión sobre la Fórmula de Radbruch aparece, tanto en escritos de Radbruch como de Alexy, puede observarse que las reflexiones se desarrollan en textos destinados de manera decisiva a la investigación del concepto de derecho. En los escritos de Radbruch, la Fórmula de Radbruch se presenta en un artículo titulado "Gesetzliches Unrecht und übergesetzliches Recht" ("Arbitraridad legal y derecho supralegal'), de 1946, esencialmente dedicado al concepto de derecho. En los textos de Alexy, aunque se presente ahí una reflexión más intensa sobre la aplicación de la Fórmula de Radbruch como una prescripción para la decisión del tribunal, el debate más profundo sobre el tema se encuentra en un libro dedicado a la investigación del concepto y la validez del derecho. ${ }^{25}$

Así, una vez considerados los escritos mismos en los que la Fórmula de Radbruch es desarrollada, tanto en textos de Radbruch como en textos de Alexy, lo que se observa es que ella es examinada no en textos destinados principalmente a la cuestión de la aplicación del derecho, sino en textos orientados hacia el problema del concepto de derecho.

25 La obra en cuestión es Begriff und Geltung des Rechts (El concepto y la validez del derecho), cuya primera edición es de 1992. 
El segundo argumento de Bix afirma que la Fórmula de Radbruch no funciona como un elemento del concepto de derecho tan pronto es interpretada desde la perspectiva de un teórico del derecho.

(...) Si la fórmula ha de entenderse como una declaración conceptual sobre la naturaleza del derecho, entonces es (por definición) un reclamo con respecto a todos los sistemas legales existentes y a todos los posibles. Esto puede no ser una buena descripción de la fórmula. Teniendo en cuenta el argumento de Radbruch de que normas significativamente injustas no son normas válidas, puede entenderse tal afirmación hecha dentro de un sistema jurídico determinado, en relación con el criterio de validez de este sistema legal. El significado de una tal declaración hecha por un teórico del derecho con respecto a todos (y a todos posibles) los sistemas jurídicos es mucho menos clara. ${ }^{26}$

El segundo argumento de Bix se basa en la distinción entre la perspectiva del observador y la perspectiva del participante. Según Bix, un teórico del derecho debe adoptar necesariamente el punto de vista del observador y, ya que la Fórmula de Radbruch - de acuerdo con Bix - no tiene sentido desde el punto de vista del observador, por tanto ella debe entonces ser tomada no como un elemento del concepto del derecho, sino como una prescripción para la decisión judicial.

El problema con este argumento es que Bix toma la perspectiva del observador como una perspectiva independiente. Esto no es correcto. La perspectiva del observador es dependiente de la perspectiva del participante (lo que significa que la perspectiva del observador no tiene sentido sin la perspectiva del participante). Ella es dependiente en dos sentidos: (1) Porque una descripción del derecho desde la perspectiva del observador presupone que existen personas que realmente observan y aplican el derecho, que es

26 Bix, Brian, «Radbruch's Formula and Conceptual Analysis», en, American Fournal of Furisprudence, 56, 2008, p. 52. 
descrito por el observador, lo cual significa que ella presupone la existencia de personas que adoptan la perspectiva del participante en relación con el derecho y (2) porque una descripción del derecho desde la perspectiva del observador asume que tanto el observador que describe el derecho como aquellos a quien esta descripción se dirige tienen la capacidad de comparar esta descripción con sus propias concepciones de derecho concebidas desde la perspectiva del participante (lo cual significa que alguien que ni siquiera sabe lo que es el derecho desde la perspectiva del participante no será capaz de entender correctamente la descripción de un comportamiento guiado por el derecho desde la perspectiva del observador).

La misma confusión en la distinción entre las perspectivas del observador y del participante aparece en las propuestas presentadas por Norbert Hoerster. Como señala Alexy, Hoerster presenta dos proposiciones en relación con esta pregunta:

Norbert Hoerster ha sostenido que, en primer lugar, existe una necesidad de contar con una designación valorativamente neutra para las leyes promulgadas conforme al ordenamiento y socialmente eficaces, como la Onceava Ordenanza, y, segundo, que no existe ninguna alternativa utilizable para la expresión "derecho". ${ }^{27}$

Al considerar estas dos afirmaciones considero que, en primer lugar, no creo que exista una necesidad de una denominación neutra en términos de valor para una norma promulgada conforme el ordenamiento y socialmente eficaz como la Onceava Ordenanza $y$, en segundo lugar, el hecho de que no exista alternativa utilizable, más allá que "derecho", para nombrar algo como la Onceava Ordenanza es, en realidad, un fuerte indicio de que esta cosa en particular (una norma promulgada conforme el ordenamiento, socialmente eficaz y valorativamente neutra) simplemente no existe (lo cual es una buena razón para que uno no se moleste en tratar de encontrar un nombre para esto, pero no una razón para aceptar

27 Alexy, Robert, op. cit., p. 56. 
darle el nombre de "derecho"). Por lo tanto, la razón por la cual creo que un término no es necesario para designar una norma promulgada de acuerdo con el ordenamiento, socialmente eficaz y valorativamente neutra, es que tal cosa no existe. El derecho no es un nombre que bien puede atribuirse a algo como la Onceava Ordenanza. Es posible usar el término "derecho" desde la perspectiva del observador, pero los límites de esta posibilidad son establecidos por la Fórmula de Radbruch. La cuestión no es si la Fórmula de Radbruch es posible desde el punto de vista del observador. La pregunta correcta es si la perspectiva del observador es posible sin la Fórmula de Radbruch y, teniendo en cuenta que la perspectiva del observador es dependiente de la perspectiva del participante, la respuesta es no.

El fundamento de las reivindicaciones de Hoerster reside en la fe que tiene en el relativismo moral. Éstas se basan en la suposición de que el relativismo moral es verdadero y, al mismo tiempo, estas mismas reivindicaciones son tratadas como un argumento a favor del relativismo moral. Esto es evidente en la centralidad de la perspectiva del observador en la interpretación de Hoerster (como también en la de Bix) a propósito de la diferencia entre los puntos de vista del observador y del participante. Pero, puesto que no hay razón para aceptar la verdad del relativismo moral, tampoco hay razón para aceptar la validez de las afirmaciones de Hoerster.

Por lo tanto, teniendo en cuenta que la distinción entre las perspectivas del observador y del participante sólo tiene sentido si la perspectiva del observador se entiende como dependiente de la perspectiva del participante, el resultado es que el argumento extraído de esta distinción no funciona como un argumento en contra de la interpretación de la Fórmula de Radbruch como elemento de una proposición conceptual sobre la naturaleza del derecho (es decir, para decirlo más concretamente, como un elemento del concepto de derecho). 


\section{v. Conclusión}

Para concluir, vuelvo a mi punto de partida: al tener en cuenta los dos enfoques básicos de la Fórmula de Radbruch, ya sea como una propuesta conceptual sobre la naturaleza del derecho y como una prescripción para la decisión judicial, cuatro combinaciones diferentes son posibles. Radbruch (de acuerdo con su interpretación tradicional) y Alexy, en el campo no-positivista, apoyan la tesis de que la Fórmula de Radbruch realiza ambas funciones. Hart, en el campo positivista, defiende la tesis que niega a la Fórmula de Radbruch tanto la función como elemento del concepto del derecho como también la de una prescripción para la decisión judicial. Bix, también en el campo positivista, niega a la Fórmula de Radbruch como elemento del concepto del derecho, pero la acepta como una prescripción para la decisión judicial. Por último, en el campo no-positivista, defiendo la tesis de que la Fórmula de Radbruch debe ser aceptada como un elemento del concepto del derecho, pero rechazada como una prescripción para la decisión judicial.

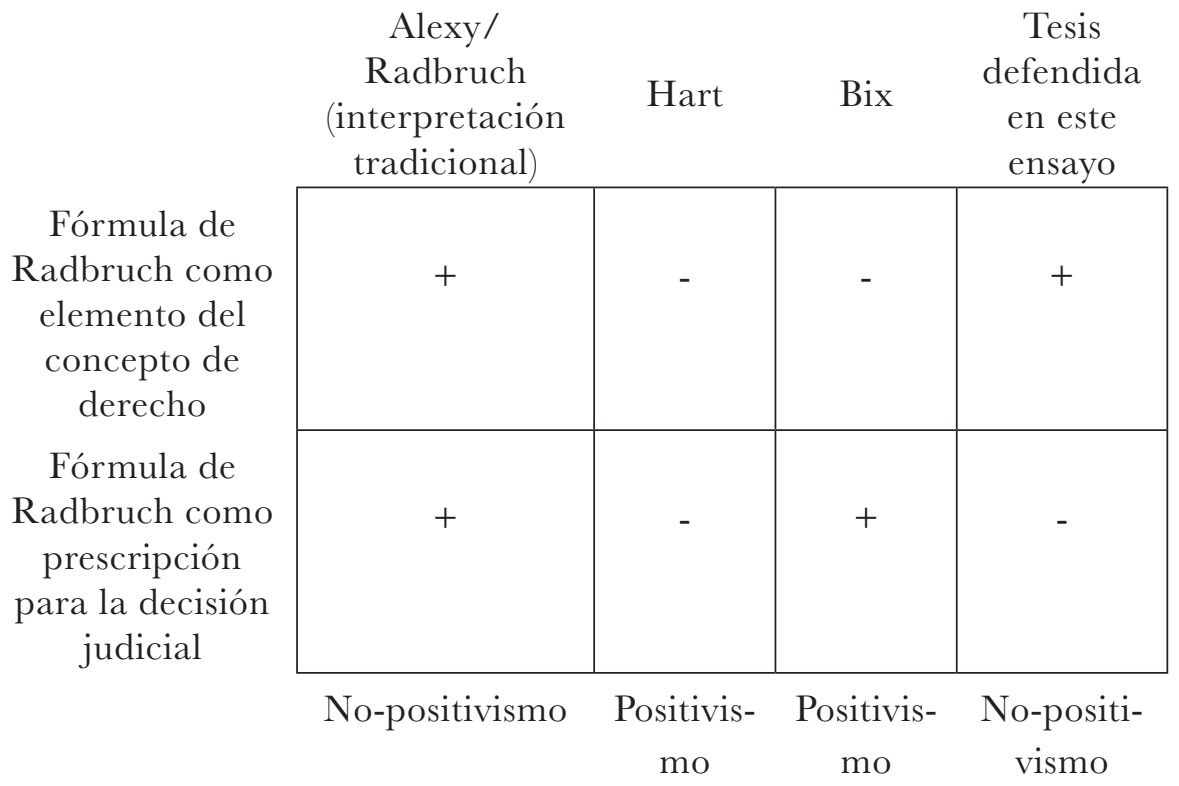


VI. BibLiografía

Alexy, Robert, Begriff und Geltung des Rechts, Múnich, Karl Alber, 1992.

_- "The Dual Nature of Law", en, Ratio Furis, Vol. 23, no. 2, 2010.

Bix, Brian, «Radbruch's Formula and Conceptual Analysis», en, American Fournal of Furisprudence, 56, 2008, pp. 45-57.

Hart, H. L. A., «Positivism and the Separation of Law and Morals», en, Harvard Law Review 71, 1957-1958, pp. 593-629.

- The Concept of Law, Oxford, Clarendon Press, 1998.

Kaufmann, Arthur, Gustav Radbruch-Rechtsdenker, Philosoph, Sozialdemokrat, Múnich, Piper, 1987.

Paulson, Stanley, L., "On the Background and Significance of Gustav Radbruch's Post-War Papers", Oxford Fournal of Legal Studies, vol. 26, No. 1 (2006): pp. 17-40.

- , "Radbruch on Unjust Laws: Competing Earlier and Later Views?", en, Oxford Journal of Legal Studies, vol. 15, No. 3, 1995: pp. 489-500.

_ 'Ein Ewiger Mythos: Gustav Radbruch als Rechtspositivist - Teil 1" Juristen-Zeitung, 2008: pp. 105-115.

_- "Lon L. Fuller, Gustav Radbruch y las tesis positivistas", en, Paulson, Stanley, L., La filosofía del derecho de Gustav Radbruch, Madrid, Marcial Pons, 2019, pp. 53-100.

Radbruch, Gustav, Rechtsphilosophie, Heidelberg, C. F. Müller, 2003.

- - "Arbitrariedad legal y derecho supralegal", en, Paulson, Stanley, L, La filosofía del derecho de Gustav Radbruch, Madrid, Marcial Pons, 2019, pp. 213-225.

Rüthers, Bernd, Die unbegrenzte Auslegung, Tubinga, Mohr Siebeck, 2012.

Wiegand, Marc, Unrichtiges Recht, Tubinga, Mohr Siebeck, 2004.

Wolf, Erik, "Umbruch oder Entwicklung in Gustav Radbruchs Rechtsphilosophie?", en, Archiv für Rechts und Sozialphilosophie 45, 1959, pp. 481-503.

Von der Pfordten, Dietmar, "Radbruch as an Affirmative Holist. On the Question of what Ought to Be Preserved of his Philosophy", Ratio Iuris, 21, 2008, pp. 387-403. 